\title{
A Family of Distributions on the Circle With Links to, and Applications Arising From, Möbius Transformation
}

\author{
S. KATO and M.C. Jones ${ }^{1}$
}

We propose a family of four-parameter distributions on the circle that contains the von Mises and wrapped Cauchy distributions as special cases. The family is derived by transforming the von Mises distribution via Möbius transformation which maps the unit circle onto itself. The densities in the family have a symmetric or asymmetric, unimodal or bimodal shape, depending on the values of parameters. Conditions for unimodality are explored. Further properties of the proposed model are obtained, many by applying the theory of the Möbius transformation. Properties of a three-parameter symmetric submodel are also investigated; these include maximum likelihood estimation, its asymptotics and a reparametrisation that proves useful quite generally. A three-parameter asymmetric subfamily, which proves adequate in each of the examples in the paper, is also discussed with emphasis on its mean direction and circular skewness. The proposed family and subfamilies are used to model an asymmetrically distributed dataset and are then adopted as the angular error distribution of a circular-circular regression model, and an application given thereof. It is in this regression context that the Möbius transformation particularly comes into its own. Comparisons with other families of circular distributions are made.

KEY WORDS: Asymmetric distributions; Circular skewness; Circular-circular regression; Unimodality; Von Mises distribution; Wrapped Cauchy distribution.

\footnotetext{
${ }^{1}$ Shogo Kato is Postdoctoral Researcher, Institute of Statistical Mathematics, 4-6-7 MinamiAzabu, Minato-ku, Tokyo, 106-8569, Japan (E-mail: skato@ism.ac.jp). M.C. Jones is Professor of Statistical Science, Department of Mathematics \& Statistics, The Open University, Milton Keynes, MK7 6AA, UK. (E-mail: m.c.jones@open.ac.uk). Kato is grateful to the Department of Mathematics \& Statistics at the Open University for its hospitality during the research visit which led to production of the paper. Financial support for the research visit was provided by the Ministry of Education, Culture, Sports, Science and Technology of Japan under a Grant-in-Aid of the 21st Century Center of Excellence for Integrative Mathematical Sciences: Progress in Mathematics Motivated by Social and Natural Sciences. The authors are extremely grateful to Dr. Arthur Pewsey for his very useful comments on the paper, some calculations, and his encouragement.
} 


\section{INTRODUCTION}

As is the case on the real line, the most familiar distributions on the circle are symmetric unimodal distributions with two parameters, one controlling location (centre of symmetry), the other scale (in this context called concentration). For example, the von Mises distribution $\operatorname{vM}(\mu, \kappa)$ has probability density function

$$
f_{V M}(\theta)=\frac{1}{2 \pi \mathcal{I}_{0}(\kappa)} \exp \{\kappa \cos (\theta-\mu)\}, \quad 0 \leq \theta<2 \pi
$$

where $0 \leq \mu<2 \pi$ is the location parameter, $\kappa \geq 0$ is the concentration parameter and $\mathcal{I}_{0}(\kappa)$ is the modified Bessel function of the first kind and order zero. This model has played a central role in directional statistics as one can see in books such as Fisher (1993), Mardia and Jupp (1999) and Jammalamadaka and SenGupta (2001). These books also cover another important distribution on the circle, the wrapped Cauchy distribution, which has density

$$
f_{W C}(\theta)=\frac{1}{2 \pi} \frac{1-r^{2}}{1+r^{2}-2 r \cos (\theta-\mu)}, \quad 0 \leq \theta<2 \pi,
$$

where $0 \leq \mu<2 \pi$ is again the location parameter and $0 \leq r<1$ is the concentration parameter. See also McCullagh (1996). Note that both the von Mises and wrapped Cauchy distributions have as special case the circular uniform distribution, when $\kappa=0$ and $r=0$, respectively.

In this article, we propose the first four-parameter extension of these distributions which allows for both asymmetry and variations in 'tailweight' encompassing both von Mises and wrapped Cauchy distributions in the extended family. The approach is one of transformation and the key notion is the important circle to circle function which is the Möbius transformation. The Möbius transformation from, say, $\tilde{\Theta}$ to $\Theta$ is given by

$$
e^{i \Theta}=e^{i \mu} \frac{e^{i \tilde{\Theta}}+r e^{i \nu}}{r e^{i(\tilde{\Theta}-\nu)}+1} \quad \text { or } \quad \Theta=\mu+\nu+2 \arctan \left[w_{r} \tan \left\{\frac{1}{2}(\tilde{\Theta}-\nu)\right\}\right]
$$

where $0 \leq \mu, \nu<2 \pi, 0 \leq r<1$, are the three parameters of the transformation ( $\mu$ controlling location) and $w_{r}=(1-r) /(1+r)$. (See Rudin, 1987, for details about the Möbius, or linear fractional, transformation.) In particular, we propose to apply 
the Möbius transformation to a random variable following the von Mises distribution; to this end, let $\tilde{\Theta}$ be distributed as $\operatorname{vM}(0, \kappa)$. Then the distribution of $\Theta$ has density given by

$f(\theta)=\frac{1-r^{2}}{2 \pi \mathcal{I}_{0}(\kappa)} \exp \left[\frac{\kappa\{\xi \cos (\theta-\eta)-2 r \cos \nu\}}{1+r^{2}-2 r \cos (\theta-\gamma)}\right] \frac{1}{1+r^{2}-2 r \cos (\theta-\gamma)}, \quad 0 \leq \theta<2 \pi$,

where $\gamma=\mu+\nu, \xi=\sqrt{r^{4}+2 r^{2} \cos (2 \nu)+1}$ and $\eta=\mu+\arg \left\{r^{2} \cos (2 \nu)+1+\right.$ $\left.i r^{2} \sin (2 \nu)\right\}$. Note that the four parameters of this distribution are $0 \leq \mu, \nu<2 \pi, 0 \leq$ $r<1$ and $\kappa>0$. (The normalizing constant in (2) can be confirmed by using (3.338.4) of Gradshteyn and Ryzhik, 1994.)

The main properties of the distribution with density (2) are explored in Section 2. These include its special cases, calculation of probabilities, closure under Möbius transformation, conditions for symmetry, conditions for unimodality, graphs of densities, skewness, a conditional derivation, random variate generation and its divergence from the wrapped Cauchy distribution. We then explore further properties of the (novel) three-parameter family of symmetric distributions associated with setting $\nu=0$ or $\pi$ in (2) in Section 3. Its probability density function, conditions for unimodality, moments and maximum likelihood estimation are investigated in Sections 3.1 to 3.4 , respectively. Note that this symmetric sub-family has a direct competitor which also covers the von Mises and wrapped Cauchy distributions, namely the distributions of Jones and Pewsey (2005); a comparison is made in Section 3.5. In Section 4, we briefly investigate an alternative, asymmetric, three-parameter subfamily of the full four-parameter model, corresponding to $\nu= \pm \pi / 2$. In particular, its density function, mean direction and skewness are studied.

Our study of the asymptotic properties of maximum likelihood estimation in Section 3.4 takes place in the context of the symmetric three-parameter special case of our full model. It includes the derivation of a reparametrisation which decreases the asymptotic correlation between two of the distribution's parameters. This reparametrisation is advocated for use in the four-parameter full model too (Section 5), although we need to resort to general optimisation methods to implement maximum likelihood estimation in all our models. Section 5 also includes a one-sample 
example where the asymmetric three-parameter special case of Section 4 turns out to be much the most suitable form of our model.

A particular merit of the current family of distributions is the way it interacts with covariates via the Möbius transformation. This has been taken advantage of for circular-circular regression with wrapped Cauchy errors by Kato, Shimizu and Shieh (2008); their work is extended to the use of family (2) in Section 6. An example involving the dependence of wind directions at midday on those in the early morning is treated there.

Unlike on the real line, on the circle it seems difficult to divorce the introduction of asymmetry from the introduction of non-unimodality. It seems desirable, however, to keep the latter to a minimum (else one runs the risk of fitting bimodal distributions to datasets that display no such bimodality, and perhaps falsely interpreting the apparent structure). In Section 2.5, and further for the symmetric case in Section 3.2 , we investigate the range of parameter values that leads to bimodality (two turning out to be the maximum number of modes possible in density (2)). In Section 7, we contrast our results (and other aspects) with properties of the four-parameter case of the generalized von Mises distribution first proposed by Maksimov (1967) and recently investigated by Gatto and Jammalamadaka (2007). Broadly speaking, we come to the conclusion that the generalized von Mises distribution is preferable if bimodality is to be expected, model (2) if not. The paper finishes with discussion in Section 8; a five-parameter extension of our distribution is among the topics considered there.

\section{PROPERTIES OF THE FOUR-PARAMETER FORM OF THE DISTRIBUTION}

\subsection{Special Cases}

The uniform distribution is transformed to the wrapped Cauchy distribution via Möbius transformation and the wrapped Cauchy is closed under that transformation (McCullagh, 1996). It follows that distribution (2) includes the von Mises $(r=0)$, wrapped Cauchy $(\kappa=0)$ and uniform $(r=\kappa=0)$ distributions as special cases. As $\kappa \rightarrow \infty$ or $r \rightarrow 1$, the model converges to a point distribution with singularity at $\theta=\mu+\nu$. 


\subsection{Probabilities}

Probabilities of intervals under density (2), i.e. quantities of the form $\int_{t_{1}}^{t_{2}} f(\theta) d \theta$, can readily be expressed in terms of similar quantities for the von Mises distribution, i.e. $\int_{s_{1}}^{s_{2}} f_{V M}(\theta) d \theta$ via the Möbius transformation (1). Specifically,

$$
s_{i}=\nu+2 \arctan \left[\frac{1}{w_{r}} \tan \left\{\frac{1}{2}\left(t_{i}-\mu-\nu\right)\right\}\right], \quad i=1,2 .
$$

This is helpful because numerical methods for calculating probabilities associated with the von Mises distribution have been developed by e.g. Hill (1977).

\subsection{Closure under Möbius Transformation}

Distribution (2) is closed under the Möbius transformation. This property is obvious from the derivation of the model and the fact that the Möbius group is closed under composition:

$$
\gamma_{2} \frac{z^{\prime}+\beta_{2}}{\bar{\beta}_{2} z^{\prime}+1}=\tilde{\gamma} \frac{z+\tilde{\beta}}{\tilde{\tilde{\beta}} z+1}, \quad|z|=1
$$

where

$z^{\prime}=\gamma_{1} \frac{z+\beta_{1}}{\bar{\beta}_{1} z+1}, \tilde{\gamma}=\gamma_{1} \gamma_{2} \frac{\overline{\gamma_{1} \beta_{1}} \beta_{2}+1}{\gamma_{1} \beta_{1} \bar{\beta}_{2}+1}, \tilde{\beta}=\bar{\gamma}_{1} \frac{\beta_{2}+\gamma_{1} \beta_{1}}{\overline{\gamma_{1} \beta_{1}} \beta_{2}+1},\left|\gamma_{j}\right|=1,\left|\beta_{j}\right|<1, j=1,2$,

(Rudin, 1987). Therefore, the distribution with density (2) is transformed into the same family with parameters $\mu, r$ and $\nu$ replaced by $\arg (\tilde{\gamma}),|\tilde{\beta}|$ and $\arg (\tilde{\beta})$, respectively. This will be particularly relevant for the regression model to follow in Section 7.

\subsection{Conditions for Symmetry}

The density (2) is, in general, asymmetrically distributed. The conditions for symmetry are given in the following theorem.

Theorem 1. The density (2) is symmetric if and only if $r=0, \nu=0, \pi$, or $\kappa=0$.

See Appendix A.1 for the proof. Of course, the symmetric special cases with $r=0$ and $\kappa=0$ are the von Mises and wrapped Cauchy distributions; the wider threeparameter family of symmetric distributions arising when $\nu=0, \pi$ will be discussed in Section 3. 


\subsection{Conditions for Unimodality}

In this subsection we discuss the modality of the proposed model (2). Without loss of generality, the location parameter $\mu$ is taken to be 0 . The first derivative of the density (2) with respect to $\theta$ is calculated as

$$
\begin{aligned}
\frac{d}{d \theta} f(\theta) \propto & \exp \left[\frac{\kappa\{\xi \cos (\theta-\eta)-2 r \cos \nu\}}{1+r^{2}-2 r \cos (\theta-\nu)}\right] \frac{1}{\left\{1+r^{2}-2 r \cos (\theta-\nu)\right\}^{3}} \\
& \times\left[-2 r \sin (\theta-\nu)\left\{1+r^{2}-2 r \cos (\theta-\nu)\right\}\right. \\
& \left.+\kappa\left(1-r^{2}\right)\left\{r^{2} \sin (\theta-2 \nu)-\sin \theta+2 r \sin \nu\right\}\right] .
\end{aligned}
$$

To discuss the modality, the problem which should be solved reduces to finding the roots of the following equation:

$$
a_{0}+a_{1} \cos \theta+a_{2} \sin \theta+a_{3} \cos \theta \sin \theta+a_{4} \cos ^{2} \theta=0
$$

where

$$
\begin{aligned}
& a_{0}=2 r\left\{r \sin (2 \nu)+\kappa\left(1-r^{2}\right) \sin \nu\right\}, \quad a_{1}=2 r\left(1+r^{2}\right) \sin \nu-\kappa r^{2}\left(1-r^{2}\right) \sin (2 \nu), \\
& a_{2}=-2 r\left(1+r^{2}\right) \cos \nu+\kappa\left(1-r^{2}\right)\left\{r^{2} \cos (2 \nu)-1\right\}, \quad a_{3}=4 r^{2} \cos (2 \nu), \\
& a_{4}=-4 r^{2} \sin (2 \nu) .
\end{aligned}
$$

As discussed in a related context by Yfantis and Borgman (1982), this equation is equivalent to a quartic equation. On putting $x=\tan (\theta / 2)$, it follows that $\cos \theta=$ $\left(1-x^{2}\right) /\left(1+x^{2}\right)$ and $\sin \theta=2 x /\left(1+x^{2}\right)$. By expressing the equation (4) as a function of $x$, we get

$$
b_{0}+b_{1} x+b_{2} x^{2}+b_{3} x^{3}+b_{4} x^{4}=0
$$

where $b_{0}=a_{0}+a_{1}+a_{4}, \quad b_{1}=2\left(a_{2}+a_{3}\right), b_{2}=2\left(a_{0}-a_{4}\right), \quad b_{3}=2\left(a_{2}-a_{3}\right), b_{4}=$ $a_{0}-a_{1}+a_{4}$. This quartic equation can be solved by using Ferrari's method as discussed in Uspensky (1948). The discriminant of the quartic equation is given by

$$
\begin{aligned}
D= & b_{1}^{2} b_{2}^{2} b_{3}^{2}-4 b_{1}^{3} b_{3}^{3}-4 b_{1}^{2} b_{2}^{3} b_{4}+18 b_{1}^{3} b_{2} b_{3} b_{4}-27 b_{1}^{4} b_{4}^{2}+256 b_{0}^{3} b_{4}^{3} \\
& +b_{0}\left(-4 b_{2}^{3} b_{3}^{2}+18 b_{1} b_{2} b_{3}^{3}+16 b_{2}^{4} b_{4}-80 b_{1} b_{2}^{2} b_{3} b_{4}-6 b_{1}^{2} b_{3}^{2} b_{4}+144 b_{1}^{2} b_{2} b_{4}^{2}\right) \\
& +b_{0}^{2}\left(-27 b_{3}^{4}+144 b_{2} b_{3}^{2} b_{4}-128 b_{2}^{2} b_{4}^{2}-192 b_{1} b_{3} b_{4}^{2}\right)
\end{aligned}
$$




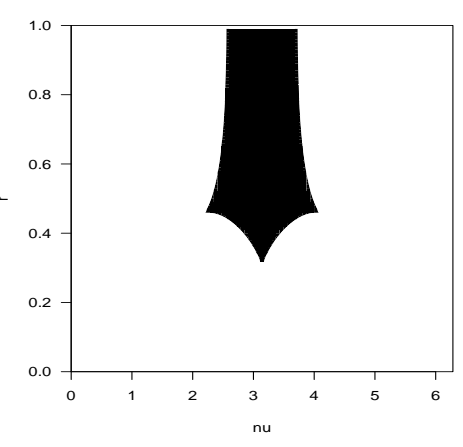

(a)

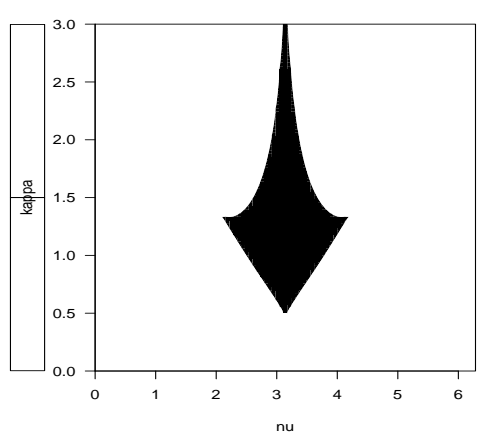

(b)

Figure 1: Discriminant (6) for density (2) as functions of: (a) $(\nu, r)$ when $\kappa=1.16$ and (b) $(\nu, \kappa)$ when $r=0.5$. The region of positive discriminant/bimodality is displayed in black while the region of negative discriminant/unimodality is exhibited in white.

It is known that equation (5) has four real roots or four complex ones if $D>0$, and two real roots and two complex ones if $D<0$. This immediately assures us that the density has no more than two modes. Moreover, the distribution is bimodal when $D>0$ and unimodal when $D<0$. Since $b_{j}, j=0, \ldots, 4$, are functions of $r, \nu$ and $\kappa$, the conditions for unimodality can, in principle, be written out in terms of these three parameters.

Figure 1 displays areas of positivity (bimodality of density) and negativity (unimodality of density) of the discriminant (6) for two pairs of parameters. For example, Figure 1(a) suggests that the density (2) with $\kappa=1.16$ can be bimodal only when, approximately, $\pi-1<\nu<\pi+1$ and $r$ is sufficiently large. (The value of $\kappa=1.16$ was chosen as the value of the von Mises concentration parameter that corresponds to the same mean resultant length as the value $r=0.5$ for the wrapped Caucchy distribution.) The frames of Figure 1 suggest that bimodality occurs for a range of values of $\nu$ around $\pi$ and large $r$ and $\kappa$.

\subsection{Graphs of Density}

In this subsection we further discuss the interpretation of the parameters by mak- 


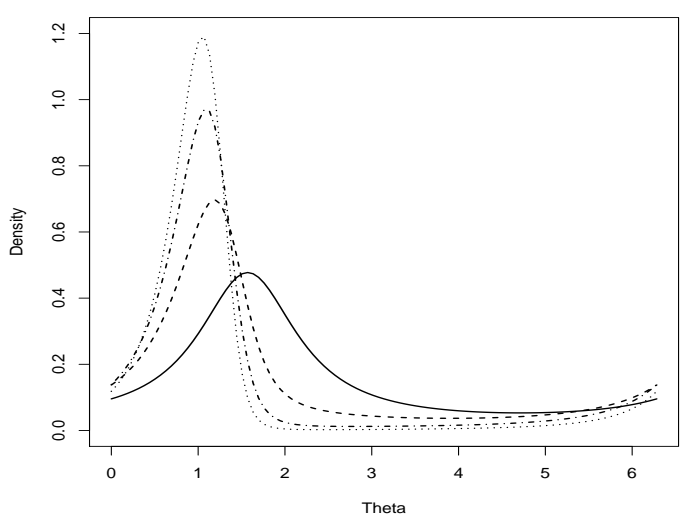

(a)

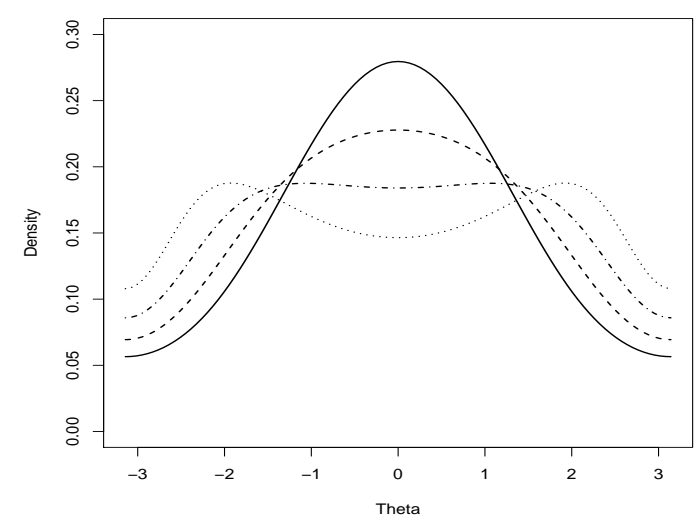

(b)

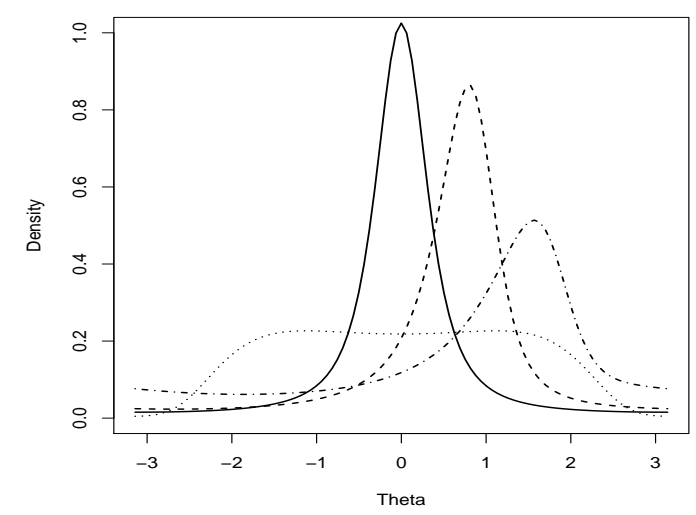

(c)

Figure 2: Density (2) for $\mu=0$ and (a) $r=0.5, \nu=\pi / 2$ and: $\kappa=0$ (solid), 1 (dashed), 2 (dot-dashed) and 3 (dotted), (b) $\kappa=1, \nu=\pi$ and: $r=0.1$ (solid), 0.2 (dashed), 0.3 (dot-dashed) and 0.4 (dotted), and (c) $\kappa=1, r=0.5$ and: $\nu=0$ (solid), $\pi / 3$ (dashed), $2 \pi / 3$ (dot-dashed) and $\pi$ (dotted). 
ing some plots of density (2) in Figure 2. As Figure 2(a) shows, the distribution generally has an asymmetric shape if $\kappa>0, r>0$ and $\nu \neq \mu+(-1)^{j} \pi(j=0,1)$. Figure 2(b) displays how the distribution changes from unimodality to bimodality as $r$ increases (in the symmetric case with $\nu=\pi$ ). Figure 2(c) exhibits the interpretation of $\nu$. This frame implies that the parameter $\nu$ has a particular role in controlling the skewness of the model; this is discussed in Sections 2.7 and 4.3.

\subsection{Skewness}

Mardia (1972) proposed a measure of skewness for circular distributions, $s$, which is defined by

$$
s=\frac{E[\sin \{2(\Theta-\alpha)\}]}{(1-\rho)^{3 / 2}},
$$

where $\alpha$ and $\rho$ are the mean direction and the mean resultant length of the distribution of $\Theta$, respectively. Here we make numerical calculations to consider how, in particular, the parameter $\nu$ influences skewness. See Figure 3. Figure 3(a) suggests that skewness increases monotonically as a function of $\nu$ when $\nu$ takes values in $[0, a]$ and decreases monotonically when $a \leq \nu \leq 2.4$ for $a \simeq 1.1$ in this case. This frame also seems to show that, in general, the greater the value of $\kappa$, the greater the absolute value of the skewness. Figure 3(b) also suggests that the skewness takes its maximum value when $\nu$ is in about $[0.8,1.6]$. We note for future reference that this interval contains the value $\pi / 2$. It seems that, as $r$ increases, the value of $\nu$ which maximises the skewness decreases.

\subsection{A Conditional Derivation}

Let $(X, Y)$ be distributed as a bivariate normal distribution $N\left(\zeta, \sigma^{2} I\right)$, where $\zeta=\left(\zeta_{1}, \zeta_{2}\right)^{\prime}$ and $I$ is the $2 \times 2$ identity matrix. Suppose $Z=X+i Y$ and $\tilde{Z}=$ $(Z+\beta) /(\bar{\beta} Z+1)$, where $|\beta| \neq 1$. It is fairly easy to see that the density of $\tilde{Z}$ is given by

$$
f(\tilde{z})=\frac{1}{2 \pi \sigma^{2}} \exp \left(-\frac{\left|\bar{\beta} \zeta_{c}+1\right|^{2}}{2 \sigma^{2}} \frac{\left|\tilde{z}-\frac{\zeta_{c}+\beta}{\bar{\beta} \zeta_{c}+1}\right|^{2}}{|1-\bar{\beta} \tilde{z}|^{2}}\right) \frac{1-|\beta|^{2}}{|1-\bar{\beta} \tilde{z}|^{2}}, \quad \tilde{z} \in \mathbb{C}
$$




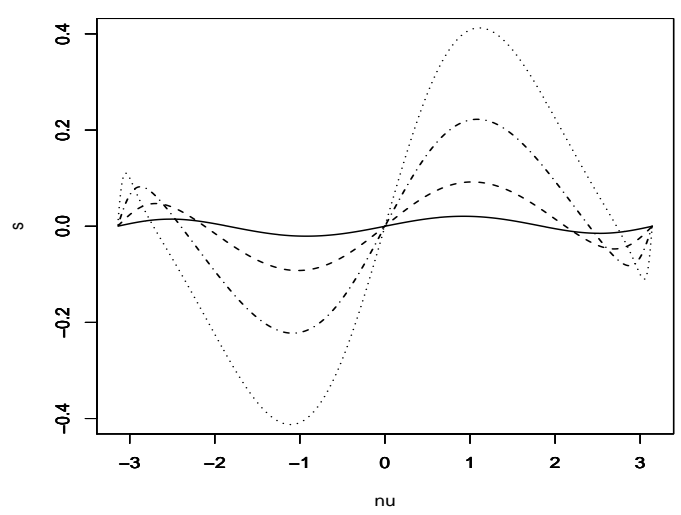

(a)

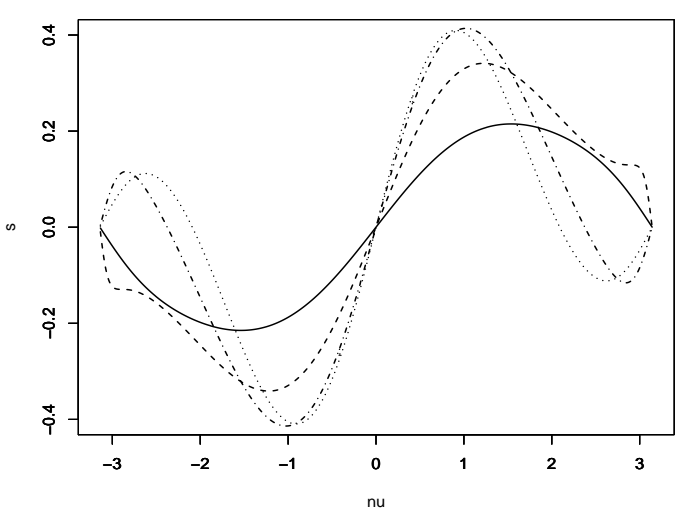

(b)

Figure 3: Skewness, $s$, for the distribution (2) as a function of $\nu$ for $\mu=0$ and: (a) $r=0.5$ and $\kappa=0.3$ (solid), 0.6 (dashed), 0.9 (dot-dashed) and 1.2 (dotted); and (b) $\kappa=1.16$ and $r=0.2$ (solid), 0.4 (dashed), 0.6 (dot-dashed) and 0.8 (dotted).

where $\zeta_{c}=\zeta_{1}+i \zeta_{2}$. Write $\tilde{Z}=\Xi e^{i \Theta}, \beta=r e^{i \nu}$ and $\zeta_{c}=\psi e^{i \mu}$. Then the density of the conditional distribution of $\Theta$ given $\Xi=\xi$ is proportional to

$$
f(\theta \mid \xi) \propto \exp \left[\frac{\kappa\left\{\cos (\theta-\eta)-\frac{\xi^{2}+\gamma^{2}}{2 \xi \gamma}\right\}}{1+r^{2} \xi^{2}-2 r \xi \cos (\theta-\nu)}\right] \frac{1}{1+r^{2} \xi^{2}-2 r \xi \cos (\theta-\nu)}, \quad 0 \leq \theta<2 \pi,
$$

where $\kappa=\xi \gamma\left\{1+r^{2} \psi^{2}+2 r \psi \cos (\mu-\nu)\right\} / \sigma^{2}$ and $\gamma e^{i \eta}=\left(\zeta_{c}+\beta\right) /\left(\bar{\beta} \zeta_{c}+1\right)$. After some reparameterization, we find that the above density is essentially the same as the density (2). This result is clear from the fact that the von Mises distribution is obtained as a conditional distribution of the bivariate normal distribution (e.g. Jammalamadaka and SenGupta, 2001, p.40) and that Möbius transformation and conditioning commute.

\subsection{Random Variate Generation}

Construction (1) affords random variate generation immediately via Möbius transformation of von Mises random variates, provided a good algorithm is available for simulating from the von Mises distribution. For example, employing the method of Best and Fisher (1979), which is an acceptance/rejection algorithm based, intrigu- 
ingly, on an envelope proportional to the wrapped Cauchy density, a random number from model (2) is generated by the following algorithm. First, set

$$
a=1+\left(1+4 \kappa^{2}\right)^{\frac{1}{2}}, \quad b=\frac{a-\sqrt{2 a}}{2 \kappa} \quad \text { and } \quad \zeta=\frac{1+b^{2}}{2 b} .
$$

Then follow the steps below:

Step 1: Generate uniform $(0,1)$ random numbers $U_{1}$ and $U_{2}$.

Step 2: Put $Z=\cos \left(\pi U_{1}\right), F=(\zeta Z+1) /(\zeta+Z)$ and $C=\kappa(\zeta-F)$.

Step 3: If $C(2-C)-U_{2}>0$, then go to Step 5 .

Step 4: If $\log \left(C / U_{2}\right)+1-C<0$, return to Step 1 .

Step 5: Generate a further uniform $(0,1)$ random number $U_{3}$ and set $\tilde{\Theta}=\operatorname{sign}\left(U_{3}-\right.$ $0.5) \times \cos ^{-1}(F)$.

Step 6: $\Theta=\mu+\nu+2 \arctan \left[\{(1-r) /(1+r)\} \tan \left\{\frac{1}{2}(\tilde{\Theta}-\nu)\right\}\right]$.

See Best and Fisher (1979, p.155) for the acceptance ratio of this algorithm.

\subsection{Divergence From the Wrapped Cauchy Distribution}

As mentioned before, the proposed model reduces to the wrapped Cauchy distribution for $\kappa=0$. For general $\kappa>0$, the density of our model is expressed as the product of that of the wrapped Cauchy, an exponential function and a Bessel function divisor. In this subsection, we discuss the distance or affinity between the proposed model and the wrapped Cauchy model by using two well-known measures of divergence, namely, those of Bhattacharyya and Kullback-Leibler.

Let $f$ and $f_{W C}$ be the densities of the proposed model (2) and the wrapped Cauchy model $\mathrm{WC}(\gamma, r)$, respectively. Then the measure of divergence given by Bhattacharyya is calculated as

$$
I^{B}\left(f, f_{W C}\right)=-\log \int_{0}^{2 \pi} \sqrt{f(\theta) f_{W C}(\theta)} d \theta
$$




$$
\begin{gathered}
=-\log \left[\frac{1-r^{2}}{2 \pi \sqrt{\mathcal{I}_{0}(\kappa)}} \int_{0}^{2 \pi} \exp \left\{\frac{\frac{1}{2} \kappa\{\xi \cos (\theta-\eta)-2 r \cos \nu\}}{1+r^{2}-2 r \cos (\theta-\gamma)}\right\}\right. \\
\left.\times \frac{d \theta}{1+r^{2}-2 r \cos (\theta-\gamma)}\right] \\
=-\log \left\{\frac{1-r^{2}}{2 \pi \sqrt{\mathcal{I}_{0}(\kappa)}} \frac{2 \pi \mathcal{I}_{0}\left(\frac{1}{2} \kappa\right)}{1-r^{2}}\right\}=-\frac{1}{2} \log \left\{\frac{\mathcal{I}_{0}^{2}\left(\frac{1}{2} \kappa\right)}{\mathcal{I}_{0}(\kappa)}\right\} .
\end{gathered}
$$

It is easy to see that $I^{B}\left(f, f_{W C}\right)=0$ when $\kappa=0$. Differentiating, we have

$$
\frac{\partial}{\partial \kappa} \frac{\mathcal{I}_{0}^{2}\left(\frac{1}{2} \kappa\right)}{\mathcal{I}_{0}(\kappa)}=\frac{\mathcal{I}_{0}\left(\frac{1}{2} \kappa\right) \mathcal{I}_{1}\left(\frac{1}{2} \kappa\right)}{\mathcal{I}_{0}(\kappa) A\left(\frac{1}{2} \kappa\right)}\left\{A\left(\frac{1}{2} \kappa\right)-A(\kappa)\right\},
$$

where $A(\kappa)=\mathcal{I}_{1}(\kappa) / \mathcal{I}_{0}(\kappa)>0$. Since $A(\kappa)$ is monotonically increasing with respect to $\kappa$ (see, for example, Jammalamadaka and SenGupta, 2001, Section 4.2.1)), it follows that $I^{B}\left(f, f_{W C}\right)$ is monotonically increasing with respect to $\kappa$.

Next, we consider directed Kullback-Leibler divergence which is defined in general as $I^{K L}\left(f_{1}, f_{2}\right)=\int_{0}^{2 \pi} \log \left\{f_{1}(\theta) / f_{2}(\theta)\right\} f_{1}(\theta) d \theta$. Applying the inverse Möbius transformation, it turns out that both $I^{K L}\left(f, f_{W C}\right)$ and $I^{K L}\left(f_{W C}, f\right)$ are readily obtainable (although, for brevity, the calculations are omitted). We have

$$
I^{K L}\left(f, f_{W C}\right)=-\log \left\{\mathcal{I}_{0}(\kappa)\right\}+\kappa A(\kappa) \quad \text { and } \quad I^{K L}\left(f_{W C}, f\right)=\log \left\{\mathcal{I}_{0}(\kappa)\right\} .
$$

Clearly, $I^{K L}\left(f, f_{W C}\right)=I^{K L}\left(f_{W C}, f\right)=0$ for $\kappa=0$. By using the fact that $A^{\prime}(\kappa)>0$ (see Jammalamadaka and SenGupta (2001, Section 4.2.1)), it follows that

$$
\frac{\partial}{\partial \kappa} I^{K L}\left(f, f_{W C}\right)=\kappa A^{\prime}(\kappa)>0 \text { and } \frac{\partial}{\partial \kappa} I^{K L}\left(f_{W C}, f\right)=A(\kappa)>0 .
$$

Hence both measures of divergence by Bhattacharyya and (both versions of directed) Kullback-Leibler show that the distance between the proposed model and the wrapped Cauchy increases as $\kappa$ increases.

\section{THE THREE-PARAMETER SYMMETRIC SPECIAL CASE}

\subsection{Probability Density Function}

In this section we investigate detailed properties of the symmetric case of the distribution (2) corresponding to $\nu=0$ or $\nu=\pi$. In this case, the density (2) reduces 
to

$f(\theta)=\frac{1-r^{2}}{2 \pi \mathcal{I}_{0}(\kappa)} \exp \left[\frac{\kappa\left\{\left(1+r^{2}\right) \cos (\theta-\mu)-2 r\right\}}{1+r^{2}-2 r \cos (\theta-\mu)}\right] \frac{1}{1+r^{2}-2 r \cos (\theta-\mu)}, \quad 0 \leq \theta<2 \pi$,

where $0 \leq \mu<2 \pi, \kappa>0$ and $-1<r<1$. Note that the domain of the parameter $r$ is extended so that it includes negative values. The distribution (7) with $r>0$ is equivalent to (2) with $\nu=0$ whereas density (7) with $r<0$ is essentially the same as density (2) with $\nu=\pi$. Clearly, this distribution is symmetric about $\theta=\mu$ and $\mu+\pi$. Of course, this model also includes the von Mises $(r=0)$, the wrapped Cauchy $(\kappa=0)$ and the uniform $(\kappa=r=0)$ distributions as special cases.

By considering the density of the rescaled random variable $\Theta^{\prime}=\mu+\sqrt{\kappa}(\Theta-\mu)$ where $\Theta \sim f$, i.e. $\kappa^{-1 / 2} f\left(\mu+\kappa^{-1 / 2}\left(\theta^{\prime}-\mu\right)\right)$, and letting $\kappa \rightarrow \infty$ we readily find that the symmetric version of our distribution tends to a normal distribution with mean $\mu$ and variance $w_{r}=(1-r) /(1+r)$. This is a generalised form of the standard result for the von Mises distribution $(r=0)$ given, for example, in Mardia and Jupp (1999, pp.37-38).

\subsection{Conditions for Unimodality}

The model (7) can be unimodal or bimodal depending on the choice of the parameters. Let $\mu=0$, without loss of generality. Then, equation (4) for this submodel can be simply expressed as

$$
\sin \theta\left\{-\kappa\left(1-r^{2}\right)^{2}-2 r\left(1+r^{2}\right)+4 r^{2} \cos \theta\right\}=0 .
$$

The condition for unimodality is thus that

$$
\text { (i) } \quad \kappa>\frac{-2 r}{(1+r)^{2}} \quad \text { or } \quad(i i) \quad \kappa<\frac{-2 r}{(1-r)^{2}} \text {. }
$$

Clearly, the model with $r>0$ is unimodal because then condition (i) holds (and it is easy to see that the mode is at 0 , and there is an antimode at $\pi$ ). The interpretation of condition (i) is that the model is unimodal because the Möbius transformation only weakly attracts the von Mises distribution towards $\pi$ (and the mode, it seems, remains at zero). On the other hand, condition (ii) yields unimodality when the 


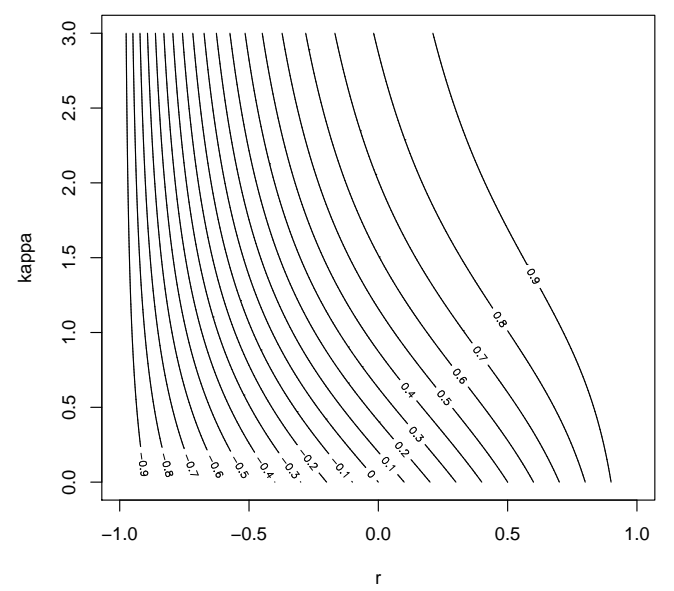

Figure 4: The first trigonometric moment of the symmetric case of distribution (2) when $\mu=\nu=0$.

Möbius transformation strongly attracts the von Mises toward $\pi$ compared with the concentration of the untransformed model (and the mode is at $\pi$ ). Bimodality occurs only in some 'in between' cases where it can be shown that the modes are at the two values of $\theta_{0}$ satisfying $\cos \theta_{0}=\left\{\kappa\left(1-r^{2}\right)^{2}+2 r\left(1+r^{2}\right)\right\} /\left(4 r^{2}\right)$.

The property of unimodality of (7) for $r \geq 0$ can be argued to be a good reason for sometimes restricting attention to the parameter domain $0 \leq r<1$.

\subsection{Moments}

The following theorem holds for the model (7). See Appendix A.2 for the proof.

Theorem 2. Let $\Theta$ have density (7) with $\mu=0$. Write $d_{r}=-4 r /(1-r)^{2}$. Then the following equation holds:

$$
\begin{aligned}
E\left(\cos ^{n} \Theta\right)= & \frac{e^{-\kappa}}{\pi \mathcal{I}_{0}(\kappa)} \sum_{j=0}^{n}\left(\begin{array}{c}
n \\
j
\end{array}\right)(-1)^{j}\left\{\frac{2\left(r^{2}+1\right)}{(1-r)^{2}}\right\}^{n-j} B\left(\frac{1}{2}+n-j, \frac{1}{2}\right) \\
& \times \Phi_{1}\left(\frac{1}{2}+n-j, n, 1+n-j, d_{r}, 2 \kappa\right),
\end{aligned}
$$

where $B(a, b)$ is the beta function and $\Phi_{1}(a, b, c, d, e)$ is the confluent hypergeometric series of two variables (Gradshteyn and Ryzhik, 1994, Section 9.26). 
Figure 4 plots the first trigonometric moment as a function of $-1<r<1$ and $\kappa>0$ when $\mu=0$. The positive and negative trigometric moments correspond to the mean direction being given by 0 and $\pi$, respectively. The figure suggests that the mean direction is 0 when $r>0$ and can be $\pi$ when $r<0$ and $\kappa$ is sufficiently small. It is also easy to see that the mean resultant length is monotonically increasing as a function of $\kappa$ when $r>0$. The contour corresponding to mean resultant length zero corresponds to $r$ and $\kappa$ satisfying

$$
\left(1-\frac{d_{r}}{2}\right) \Phi_{1}\left(\frac{3}{2}, 1,2, d_{r}, 2 \kappa\right)=\Phi_{1}\left(\frac{1}{2}, 1,1, d_{r}, 2 \kappa\right)
$$

\subsection{Maximum Likelihood Estimation}

Consider an i.i.d. sample $\theta_{1}, \ldots, \theta_{n}$ from density (7) and let the log-likelihood function be denoted by $\ell$. By way of notation, write

$$
B_{i}=B_{i}(\mu, r)=1+r^{2}-2 r \cos \left(\theta_{i}-\mu\right)
$$

Differentiation reveals elements of the observed information matrix to be

$$
\begin{gathered}
-\frac{\partial^{2} \ell}{\partial \mu^{2}}=\sum_{i=1}^{n}\left[\left\{\frac{\kappa\left(1-r^{2}\right)^{2}}{B_{i}^{2}}+\frac{2 r}{B_{i}}\right\} \cos \left(\theta_{i}-\mu\right)-4 r\left\{\frac{\kappa\left(1-r^{2}\right)^{2}}{B_{i}^{3}}+\frac{r}{B_{i}^{2}}\right\} \sin ^{2}\left(\theta_{i}-\mu\right)\right] \\
-\frac{\partial^{2} \ell}{\partial \mu \partial r}=-2 \sum_{i=1}^{n}\left[\frac{2 \kappa\left\{\xi \cos \left(\theta_{i}-\eta\right)-2 r \cos \nu\right\}}{B_{i}^{3}}+\frac{1}{B_{i}^{2}}\right]\left(1-r^{2}\right) \sin \left(\theta_{i}-\mu\right) \\
-\frac{\partial^{2} \ell}{\partial \mu \partial \kappa}=-\left(1-r^{2}\right)^{2} \sum_{i=1}^{n} \frac{\sin \left(\theta_{i}-\mu\right)}{B_{i}^{2}} \\
-\frac{\partial^{2} \ell}{\partial r^{2}}=2\left[\frac{n\left(1+r^{2}\right)}{\left(1-r^{2}\right)^{2}}+\sum_{i=1}^{n}\left\{\frac{\sin ^{2}\left(\theta_{i}-\mu\right)-\left(r-\cos \left(\theta_{i}-\mu\right)\right)^{2}}{B_{i}^{2}}\right.\right. \\
\left.\left.+\quad 2 \kappa \frac{\left\{2 \cos \left(\theta_{i}-\mu\right)-3 r+r^{3}\right\}}{B_{i}^{3}} \sin ^{2}\left(\theta_{i}-\mu\right)\right\}\right] \\
-\frac{\partial^{2} \ell}{\partial r \partial \kappa}=2\left(1-r^{2}\right) \sum_{i=1}^{n} \frac{\sin ^{2}\left(\theta_{i}-\mu\right)}{B_{i}^{2}} \\
-\frac{\partial^{2} \ell}{\partial \kappa^{2}}=n\left(1-\frac{\mathcal{I}_{1}^{2}(\kappa)}{\mathcal{I}_{0}^{2}(\kappa)}-\frac{1}{\kappa} \frac{\mathcal{I}_{1}(\kappa)}{\mathcal{I}_{0}(\kappa)}\right)
\end{gathered}
$$




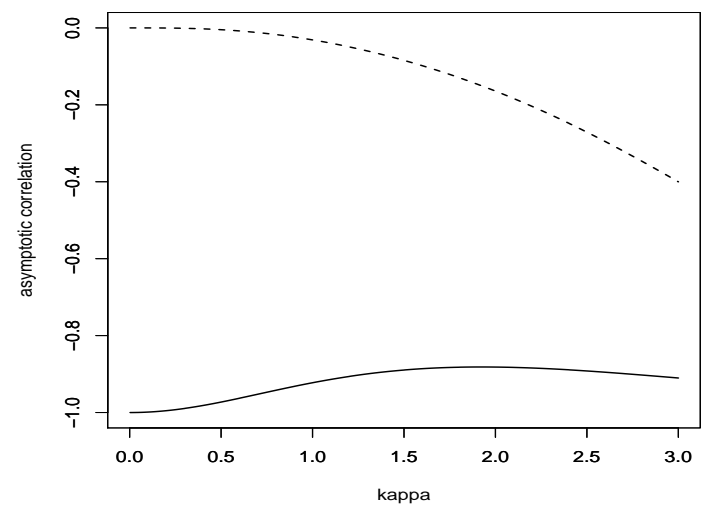

Figure 5: Plot of asymptotic correlations between $\hat{\kappa}$ and $\hat{r}$ (solid line) and between $\hat{\kappa}$ and $\hat{s}$ (dashed line).

Now consider elements of the expected information matrix, $\iota_{\alpha, \beta} \equiv E\left\{-\partial^{2} \ell / \partial \alpha \partial \beta\right\}$. Attractively,

$$
\iota_{\mu, r}=\iota_{\mu, \kappa}=0
$$

so that $\hat{\mu}$ is asymptotically independent of $\hat{r}$ and $\hat{\kappa}$ (where the 'hats' denote maximum likelihood estimators); this is a consequence of $\mu$ being the location parameter of a symmetric distribution. Perhaps surprisingly, the necessary expectations can be explicitly performed to obtain helpful formulae for the other elements of the expected information matrix; see Appendix A.3 for an indication of these calculations. We now concentrate on the elements associated with $r$ and $\kappa$; they turn out to be

$$
\iota_{r, r}=\frac{2 n}{\left(1-r^{2}\right)^{2}}\left\{1+3 \frac{\mathcal{I}_{2}(\kappa)}{\mathcal{I}_{0}(\kappa)}\right\} \quad \text { and } \quad \iota_{r, \kappa}=\frac{n}{\left(1-r^{2}\right)}\left\{1-\frac{\mathcal{I}_{2}(\kappa)}{\mathcal{I}_{0}(\kappa)}\right\}
$$

while $\iota_{\kappa, \kappa}$ is, of course, given by the formula in (10).

Note that $\iota_{r, \kappa}>0$ which means that the asymptotic correlation between $\hat{r}$ and $\hat{\kappa}$, given by

$$
-\frac{\iota_{r, \kappa}}{\sqrt{\iota_{r, r} \iota_{\kappa, \kappa}}}=\frac{\sqrt{\mathcal{I}_{0}(\kappa)}\left(\mathcal{I}_{2}(\kappa)-\mathcal{I}_{0}(\kappa)\right)}{\left(2\left\{\mathcal{I}_{0}^{2}(\kappa)-\mathcal{I}_{1}^{2}(\kappa)-\kappa^{-1} \mathcal{I}_{0}(\kappa) \mathcal{I}_{1}(\kappa)\right\}\left[\mathcal{I}_{0}(\kappa)-\mathcal{I}_{2}(\kappa)+\kappa\left\{\mathcal{I}_{1}(\kappa)-\mathcal{I}_{3}(\kappa)\right\}\right]\right)^{1 / 2}}
$$

is negative. It is also, remarkably, independent of the value of $r$. However, as is typical of many three-parameter symmetric models, the absolute value of the asymptotic correlation is very high: see the lower curve in Figure 5. 
We can, however, take advantage of the special structure of the expected Fisher information matrix to attempt construction of an orthogonal reparametrisation (Cox and Reid, 1987). In particular, we can write

$$
n^{-1} \iota_{r, r}=T^{2}(r) S(\kappa), \quad n^{-1} \iota_{r, \kappa}=T(r) C(\kappa) \text { and } n^{-1} \iota_{\kappa, \kappa}=J(\kappa)
$$

for appropriate functions of $r$ and $\kappa$ only. Then consider a reparametrisation of the form $\{s, \kappa\}=\{s(r, \kappa), \kappa\}$. Write $s^{i j}(r, \kappa)=\partial^{i+j} s(r, \kappa) / \partial r^{i} \partial \kappa^{j}, i, j=0,1$. The asymptotic correlation between $\hat{s}$ and $\hat{\kappa}$ is proportional to

$$
s^{01}(r, \kappa) \iota_{r, r}-s^{10}(r, \kappa) \iota_{r, \kappa} \propto s^{01}(r, \kappa) T(r) S(\kappa)-s^{10}(r, \kappa) C(\kappa)
$$

This is zero if we take $s(r, \kappa)=A(r) B(\kappa)$ where $\partial \log A(r) / \partial r=T(r)$ and $\partial \log B(\kappa) / \partial \kappa=$ $C(\kappa) / S(\kappa)$. Since $T(r)=\left(1-r^{2}\right)^{-1}$, this yields an explicit form for $A(r)$ which is $\sqrt{(1+r) /(1-r)}$. No such explicit result is forthcoming for $B(\kappa)$ so we resort to a small $\kappa$ approximation which yields small correlation for small $\kappa$ and, hopefully, does not increase too rapidly for larger $\kappa$. We investigated several versions of this asymptotic approximation, much the best of which turned out to be that associated with $C(\kappa) / S(\kappa) \simeq\left(2+\kappa^{2}\right)^{-1}$ leading to $B(\kappa) \simeq \exp \left(\tan ^{-1}(\kappa / \sqrt{2}) / \sqrt{2}\right)$. The much smaller asymptotic correlation between

$$
s(\hat{r}, \hat{\kappa})=\sqrt{\frac{1+\hat{r}}{1-\hat{r}}} \exp \left\{\frac{1}{\sqrt{2}} \tan ^{-1}\left(\frac{\hat{\kappa}}{\sqrt{2}}\right)\right\}
$$

and $\hat{\kappa}$ is portrayed by the upper curve in Figure 5. (We also looked at replacing $\kappa$ rather than $r$ by $s(r, \kappa)$ but to less effect.)

A helpful knock-on effect of the reparametrisation above is that the asymptotic variance of $\hat{\kappa}$ is much reduced: see Figure 6. Reparametrisation (11) seems to us to be useful in general practice; see Section 5 .

\subsection{Comparison With the Jones and Pewsey Distribution}

An alternative three-parameter family of symmetric circular distributions was proposed by Jones and Pewsey (2005). This family of distributions has density

$$
f_{J P}(\theta)=\frac{\{\cosh (\kappa \psi)+\sinh (\kappa \psi) \cos (\theta-\mu)\}^{1 / \psi}}{2 \pi P_{1 / \psi}(\cosh (\kappa \psi))}, \quad 0 \leq \theta<2 \pi,
$$




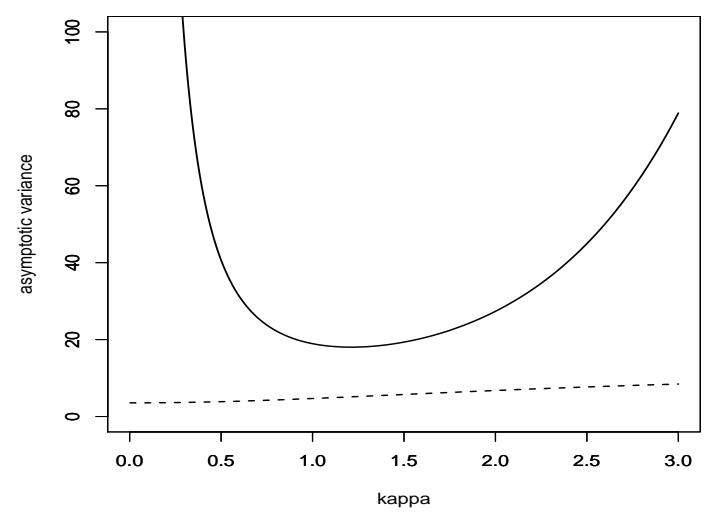

Figure 6: Plot of asymptotic variances of $\hat{\kappa}$ for the original parametrisation (solid line) and the new parametrisation (dashed line).

where $P_{1 / \psi}(z)$ is the associated Legendre function of the first kind of degree $1 / \psi$ and order 0 (Gradshteyn and Ryzhik, 1994, Sections 8.7, 8.8) and, again, $0 \leq \mu<2 \pi$ is a location parameter, $\kappa>0$ and $-\infty<\psi<\infty$. Distributions (12) and (7) have several areas of commonality and, where they differ, are somewhat complementary.

Both (12) and (7) are:

- symmetric about $\theta=\mu$ and $\mu+\pi$;

- unimodal with mode at $\theta=\mu$ and antimode at $\mu+\pi$ (provided we take $r \geq 0$ in the case of $(7))$;

- have the von Mises and wrapped Cauchy distributions as special cases (when $\psi=0$ and $\psi=-1$, respectively, in (12));

- have a similar level of complexity (normalising constant, trigonometric moments, etc);

- are of similar nature in terms of maximum likelihood estimation;

- arise from conditioning distributions associated with the bivariate normal distribution in different ways.

An advantage of the Jones and Pewsey (2005) distribution is:

- it includes a wider range of pre-considered special case densities, including the 
cardioid and Cartwright's power-of-cosine distribution.

Advantages of distribution (7) include:

- its attractive properties vis-a-vis the Möbius transformation;

- its ready extension to a four-parameter asymmetric family of distributions.

Note that Jones and Pewsey (2005, p.1422) also give a brief comparison of their distribution with the (three-parameter) family of wrapped symmetric stable distributions (Mardia, 1972, Section 3.4.8e, Jammalamadaka and SenGupta, 2001, Section 2.2.8, Gatto and Jammalamadaka, 2003). This distribution is very similar to the wrapped $t$ distribution (Pewsey, Lewis and Jones, 2007).

\section{A THREE-PARAMETER ASYMMETRIC SPECIAL CASE}

In building up four-parameter families of distributions on the real line, one sometimes takes a route through a three-parameter subfamily where the third parameter controls tailweight while the distributions retain symmetry; such a subfamily of (2) has, of course, just been considered in Section 3. Perhaps more often, however, the first introduction is that of a third parameter controlling skewness. It would be helpful if such a three-parameter asymmetric subfamily of (2) were also available. To this end, in this section, partly building on the skewness insights of Section 2.7 and partly on grounds of simplicity, we briefly investigate the asymmetric models associated with $\nu= \pm \pi / 2$.

\subsection{Probability Density Function}

In this case density (2) reduces to

$$
f(\theta)=\frac{1-r^{2}}{2 \pi \mathcal{I}_{0}(\kappa)} \exp \left\{\frac{\kappa\left(1-r^{2}\right) \cos (\theta-\mu)}{1+r^{2}-2 r \sin (\theta-\mu)}\right\} \frac{1}{1+r^{2}-2 r \sin (\theta-\mu)}, \quad 0 \leq \theta<2 \pi,
$$

where $r$ is redefined such that $-1<r<1$ and the other parameters are defined in the same manner as in (2). Clearly, $f(\mu+\theta ; \mu, r, \nu)=f(\mu-\theta ; \mu,-r, \nu)$ for any $\theta$. Plots of density (13) for specified values of the parameters can be seen in Figure 1(a). As this frame shows, this model is asymmetric except for the special cases $r=0$ and $\kappa=0$ and, in the cases shown at least, remains unimodal. 


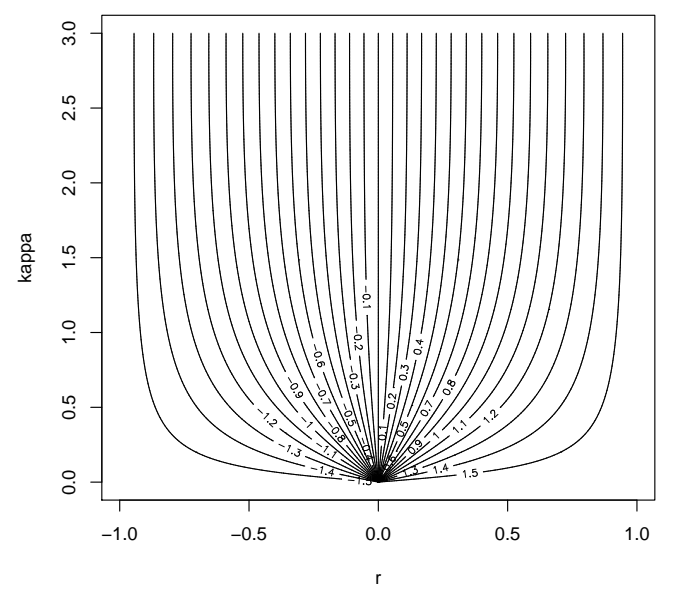

Figure 7: Contour plot of mean direction of the three-parameter asymmetric submodel (13) with $\mu=0$ as a function of $r$ and $\kappa$.

\subsection{Mean Direction}

We briefly discuss how the parameters, $r$ and $\kappa$, determine the mean direction of the three-parameter asymmetric submodel. A contour plot of the mean direction for this submodel with $\mu=0$ is displayed in Figure 7. This figure implies that, for small $\kappa$, the mean direction tends to $\pm \frac{1}{2} \pi$ at high speed as $|r|$ moves away from 0 . On the other hand, for sufficiently large $\kappa$, the mean direction depends mainly on $r$, with $\kappa$ having little influence.

\subsection{Skewness}

As one can see in Figure 3, the absolute value of skewness for the general model (2) is relatively large when $\nu= \pm \pi / 2$. Figure 8 plots skewness for the proposed model (13) for specified values of its parameters. In Figure 8(a), skewness is plotted as a function $\kappa$. This frame suggests that skewness is monotonically increasing as a function of $\kappa$ when $\kappa \leq 3$. Figure $8(\mathrm{~b})$ displays skewness as a function of $r$. It seems that the curves in this frame show concavity. This frame also shows that as $r$ approaches 0 or 1 , the skewness tends to zero. 


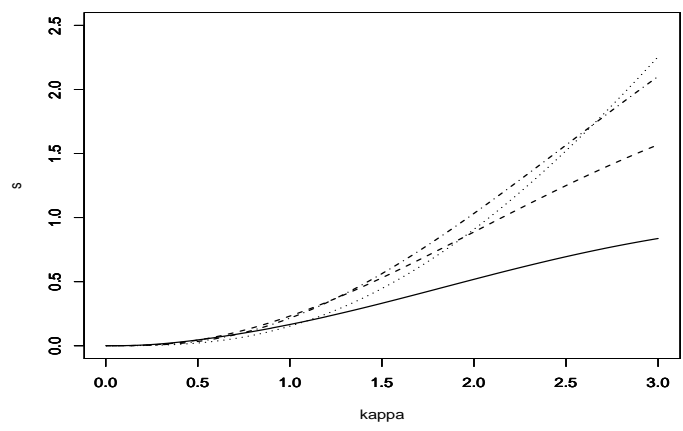

(a)

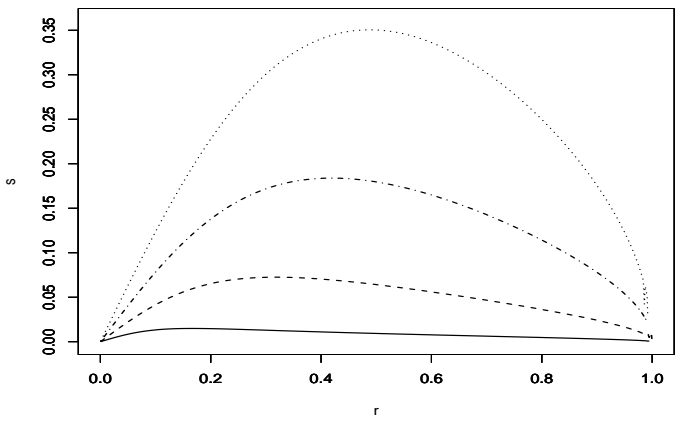

(b)

Figure 8: Skewness, $s$, for the distribution (13) with $\mu=0$ as a function of: (a) $\kappa$ for $r=0.2$ (solid), 0.4 (dashed), 0.6 (dot-dashed) and 0.8 (dotted); and (b) $r$ for $\kappa=0.3$ (solid), 0.6(dashed), 0.9 (dot-dashed) and 1.2 (dotted).

\section{MAXIMUM LIKELIHOOD ESTIMATION AND ONE-SAMPLE EXAMPLE}

Consider an i.i.d. sample $\theta_{1}, \ldots, \theta_{n}$ from density (2) and let the log-likelihood function be denoted by $\ell$. We do not write out the general score equations here because they are not especially informative or useful. For instance, no explicit formulae for maximum likelihood estimates (mle's) arise nor do the score equations give rise to any simple iterative method of solution. (This is true of the mle's for the symmetric case in Section 3.4 also.) It seems that we cannot guarantee uniqueness of the mle. In our example, we resort to direct maximisation of $\ell$ with multiple restarts to check for multiple maxima. The work of Section 3.4 is informative, however, in suggesting a reparametrisation which we employ in the general case also. We are, therefore, maximising $\ell$ over the parameter set $\{\mu, \nu, s, \kappa\}$.

As an illustrative example, we consider a dataset of azimuths of cross-beds in the rocks of the upper Kamthi river valley, India (SenGupta and Rao, 1966, Mardia and Jupp, 1999, Table 1.3; $n=580$ ); Mardia and Jupp present the data (but do not analyse them) as "an example of an asymmetrical distribution" on the circle. We fit the full model (2) and some submodels to the dataset based on the method of maximum likelihood, and compare the results. Note that the data were provided in 
histogram form (with bins of width $20^{\circ}$ ) and this discretisation is taken into account in our likelihood, making use of the approach to calculating probabilities (here, over bins) described in Section 2.2.

Table 1. Maximum Likelihood Estimates of the Parameters, the Maximised Log-Likelihood, AIC and BIC Values for the Full Model (2) and Four of its Sub-Models.

\begin{tabular}{c|ccccccc} 
Model & $\hat{\kappa}$ & $\hat{r}$ & $\hat{\nu}$ & $\hat{\mu}$ & $\log L$ & AIC & BIC \\
\hline full model (2) & 1.93 & 0.130 & 1.87 & 5.74 & -1380.59 & 2769.18 & 2786.63 \\
3-parameter asymmetric, (13) & 1.78 & 0.122 & $\left(\frac{\pi}{2}\right)$ & 5.76 & -1380.80 & 2767.60 & 2780.69 \\
3-parameter symmetric, (7) & 1.66 & 0.0428 & $(0)$ & 5.99 & -1385.41 & 2776.82 & 2789.92 \\
von Mises & 1.81 & $(0)$ & $(0)$ & 5.98 & -1385.68 & 2775.36 & 2784.08 \\
wrapped Cauchy & $(0)$ & 0.586 & $(0)$ & 6.02 & -1403.72 & 2811.44 & 2820.16
\end{tabular}

Table 1 shows maximum likelihood estimates of the parameters (converted back to the original parametrisation), the maximised log-likelihood, AIC and BIC values for the full model (2), the three-parameter asymmetric submodel (13), the threeparameter symmetric submodel (7), the von Mises and wrapped Cauchy distributions. Notice that the fitted three-parameter symmetric submodel reduces, essentially, to the von Mises distribution. However, by comparing the maximised log-likelihoods, we find that there is no support for any of the latter three, symmetric, models. The maximised log-likelihoods associated with the full model (2) and the three-parameter asymmetric submodel (13) are, however, close. Applying the likelihood ratio test to test $H_{0}: \quad \nu=\pi / 2$ against $H_{1}: \quad \nu \neq \pi / 2$ in model (2), the test statistic is $t=-2\{-1380.80-(-1380.59)\}=0.42$ with $P(T>0.42) \approx 0.517$ under the $\chi_{1}^{2}$ distribution, so this provides no evidence to reject the null hypothesis. According to the AIC and BIC criteria, which also take the simplicity of the model into account, the three-parameter asymmetric submodel is also most reasonable among the five models given in the table. (The second and third best models swap places according to whether AIC or BIC is considered.) 


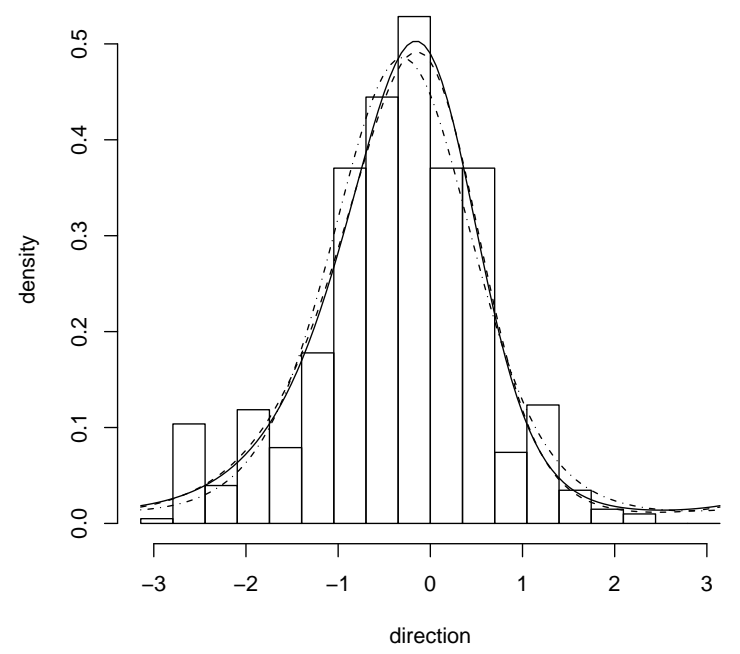

Figure 9: Histogram of the data with the maximum likelihood fits of the full family of distributions (7) (dashed), the three-parameter asymmetric submodel (13) (solid) and the von Mises distribution (dot-dashed).

Figure 9 plots the histogram of the data and the maximum likelihood fitted densities of the full model (2), the three-parameter asymmetric submodel (13) and the von Mises distribution. The similarity of the fits of models (2) and (13) is clear, as is the fact that the dataset is indeed (mildly) asymmetrically distributed. At one level, and for some purposes, it can be pronounced (see Figure 9) that this dataset can be well modelled by distribution (13) which achieves its good fit using just three parameters (and which does not introduce what would here be an inappropriate bimodality into the model). That said, chi-squared goodness-of-fit tests resoundingly reject the fit of this model (and, of course, its submodels). This is because, at a more detailed level, the dataset appears to display a degree of multimodality.

\section{CIRCULAR-CIRCULAR REGRESSION}

We consider pairs of wind directions measured at a weather station in Texas. The data we discuss here are part of a larger dataset which is taken from the Codiac data archive and is available at http://data. eol .ucar .edu/codiac/dss/id=85.034. The 
full dataset contains hourly resolution surface meteorological data from the Texas Natural Resources Conservation Commission Air Quality Monitoring Network, from May 20 to July 31, inclusive, 2003. (These data are provided by NCAR/EOL under the sponsorship of the National Science Foundation.) We look at just the 73 pairs of wind directions at 6 a.m. and noon measured each day at a particular weather station which is denoted by C28_1 in the dataset. Figure 10(a) shows a planar plot of the data; there is clearly some dependence between the wind directions at 6 a.m. and at noon.

We use a regression model which adopts model (2) as angular error distribution to regress the wind direction at noon on that at 6 a.m., and compare its fit with existing regression models. Let $Y_{j}$ and $x_{j}(1 \leq j \leq 73)$ be the response (wind direction at noon) and the covariate (wind direction at 6 a.m.), respectively. Then the extended regression model we discuss here is given by

$$
Y_{j}=\beta_{0} \frac{x_{j}+\beta_{1}}{1+\bar{\beta}_{1} x_{j}} \varepsilon_{j}, \quad\left|x_{j}\right|=1,
$$

where $\left|\beta_{0}\right|=1, \beta_{1} \in \mathbb{C}$ and $\left\{\arg \left(\varepsilon_{j}\right)\right\}$ are independent and distributed as model (2) with mean direction 0, i.e., $\mu=-\arg \left\{\int_{0}^{2 \pi} e^{i \theta} f(\theta ; \kappa, r, \nu, \mu=0) d \theta\right\}$. This model is an extension of the regression models of Downs and Mardia (2002) and Kato, Shimizu and Shieh (2008), which use the regression curve given in (14) and adopt the von Mises and wrapped Cauchy distributions, respectively, for the angular errors. Since we do not desire bimodality for the angular error distribution, we make a restriction on the parameter space, namely, assume that $\nu \in\left[-\frac{\pi}{2}, \frac{\pi}{2}\right]$. This restriction enables us to avoid bimodality except when both $\kappa$ and $r$ take large values, while describing a sufficiently wide range of skewness as seen in Figure 3 and including the threeparameter symmetric and asymmetric submodels.

To estimate the parameters based on maximum likelihood, we adopt an optimization method, the PORT routine, which can be carried out using the command nlminb in $\mathrm{R}$.

Table 2 shows the maximum likelihood estimates, the maximised log-likelihood, AIC and BIC values for the extended model (14), along with those of its von Mises 
and wrapped Cauchy special cases. According to the AIC criterion and a comparison of log-likelihoods, the extended model (14) is required to provide the most reasonable fit among the three models. The BIC criterion, which penalises a greater number of parameters more strongly, disagrees. Note that it happens that the estimated angular error distribution reduces to the three-parameter asymmetric submodel (13) since $\hat{\nu}=-\frac{\pi}{2}$. BIC for this model is 210.8 which is lower than that for the simpler alternatives. In addition to the planar plot of the data set, Figure 10(a) also shows the estimated regression curve which seems to describe the relationship between paired wind directions reasonably well.

Table 2. Maximum likelihood estimates of the parameters, the maximised log-likelihood, AIC and BIC values for the extended model (14) and two of its sub-models.

\begin{tabular}{c|cccccccccc} 
Model & $\arg \left(\hat{\beta}_{0}\right)$ & $\left|\hat{\beta}_{1}\right|$ & $\arg \left(\hat{\beta}_{1}\right)$ & $\hat{\kappa}$ & $\hat{r}$ & $\hat{\nu}$ & $\hat{\mu}$ & $\log L$ & $\mathrm{AIC}$ & $\mathrm{BIC}$ \\
\hline model (14) & 0.317 & 0.255 & -0.558 & 1.40 & 0.399 & -1.57 & $(0.772)$ & -94.7 & 201.4 & 215.1 \\
von Mises & 0.280 & 0.384 & -0.498 & 1.79 & $(0)$ & $(-)$ & $(0)$ & -97.5 & 203.1 & 212.2 \\
wrapped Cauchy & 0.216 & 0.299 & -0.470 & $(0)$ & 0.609 & $(0)$ & $(0)$ & -97.7 & 203.5 & 212.6
\end{tabular}

Next we conduct a residual analysis. Figure 10(b) exhibits a histogram of the residuals expressed in terms of radians, i.e. $\left\{\arg \left(y_{j} \bar{\beta}_{0}\left(\bar{x}_{j}+\bar{\beta}_{1}\right) /\left(1+\beta_{1} \bar{x}_{j}\right)\right)\right\}$, and the fitted density (2). From this frame, it appears that the residuals are asymmetrically distributed. However, the large-sample test of circular symmetry of Pewsey (2002) leads to the statistic $T=\bar{b}_{2} / \sqrt{\operatorname{vâr}\left(\bar{b}_{2}\right)}=-1.72$ with $P(|T| \geq 1.72) \simeq 0.0856$; similar $p$-values arise from bootstrap and randomization versions of the test. The assumption of symmetry is not rejected at the usual levels, albeit somewhat marginally. The estimated (asymmetric) density shown in the same frame shows a satisfactory visual fit to the residuals. Circular distances between the responses $y$ and predictors $\hat{y}$ are plotted in Figure 10(c). Here, the circular distance is defined by $d(y, \hat{y})=1-$ $\cos (y-\hat{y})$. Judging from the frame, the fit of the extended model seems reasonably satisfactory. The points predicted least well by the regression model are the two nearest the top left corner of Figure 10(a), the two apparently 'between' but furthest 


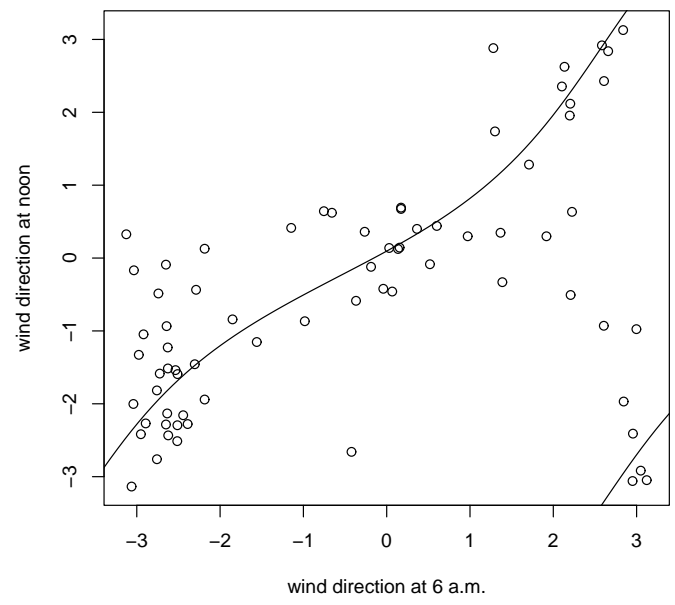

(a)

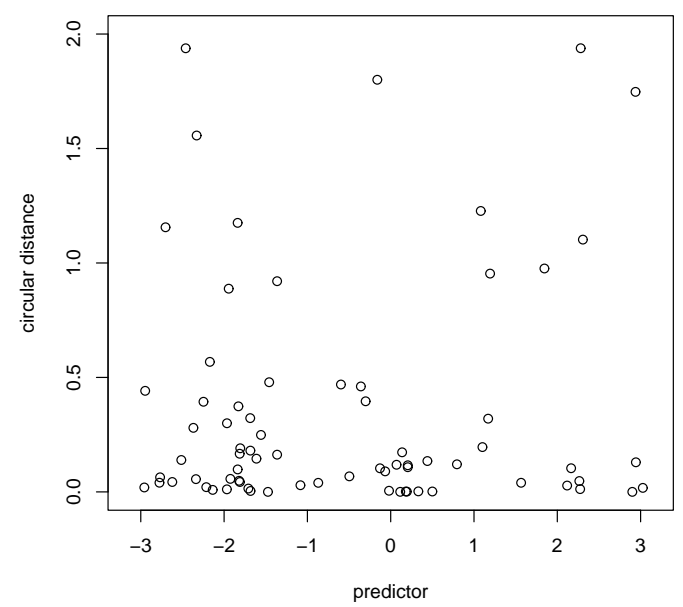

(c)

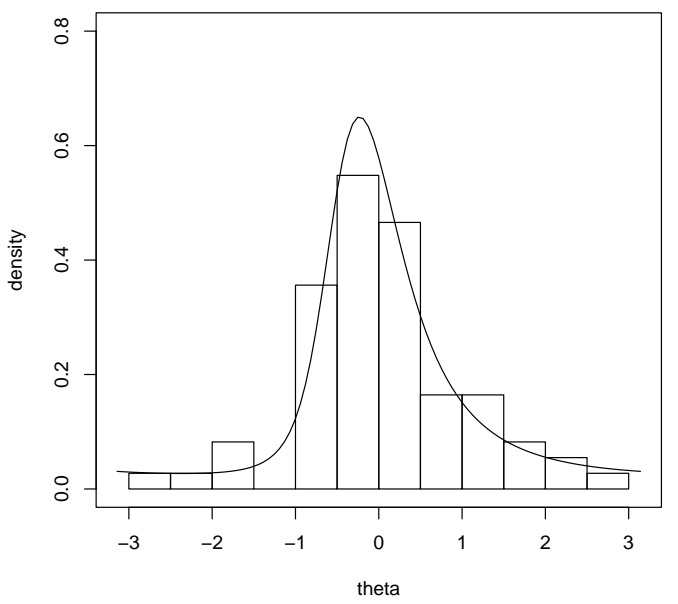

(b)

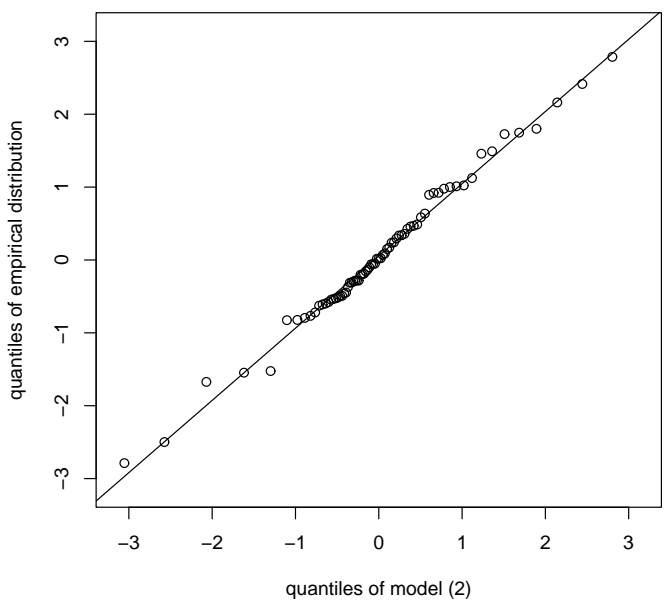

(d)

Figure 10: (a) planar plot of the wind directions at 6 a.m. and noon with the estimated regression curve, (b) histogram of the residuals and the fitted density, (c) circular distances between the response (wind direction at noon) and predicted values and (d) Q-Q plot for the extended model (14) where quantiles of the angular error distribution (x-axis) and of the empirical error distribution (y-axis) are plotted. 
from the regression line towards the right-hand side of this frame and the point isolated middle-bottom of the frame. The goodness of fit of the model is confirmed in Figure 10(d) displaying a Q-Q plot for model (14), which is a plot of quantiles of the angular error distribution against those of the empirical error distribution.

\section{COMPARISON WITH THE GENERALIZED VON MISES DISTRIBUTION}

The generalized von Mises (henceforth gvM) distribution of Maksimov (1967) (see also Yfantis and Borgman, 1982, and especially Gatto and Jammalamadaka, 2007) has, for its four-parameter special case, density proportional to

$$
\exp \left[\kappa_{1} \cos \left(\theta-\mu_{1}\right)+\kappa_{2} \cos \left\{2\left(\theta-\mu_{2}\right)\right\}\right], \quad 0 \leq \theta<2 \pi
$$

$0 \leq \mu_{1}<2 \pi, 0 \leq \mu_{2}<\pi, \kappa_{1}, \kappa_{2}>0$. Properties in common with distribution (2) include:

- symmetric and asymmetric distributions;

- being unimodal or at most bimodal;

- having the von Mises distribution as a special case;

- arising from the bivariate normal distribution (in the generalized von Mises case, directly) by a conditioning argument.

A particular advantage of the gvM distribution is its membership of the exponential family and hence a number of nice inferential properties ensue. In particular, the gvM distribution has the advantage over distribution (2) of having a concave log-

likelihood function and hence a unique maximum likelihood estimator. (Gatto and Jammalamadaka, 2007, also emphasise an entropy maximisation property of the gvM distribution.)

On the other hand, the normalising constant associated with the gvM distribution is an infinite sum of products of modified Bessel functions. Hence, trigonometric moments are not readily available either. The gvM distribution has no particular link with the wrapped Cauchy distribution (or the Möbius transformation).

The gvM distribution turns out to display bimodality for a much greater range of parameter values than does the current proposal. To see this, note that Gatto 


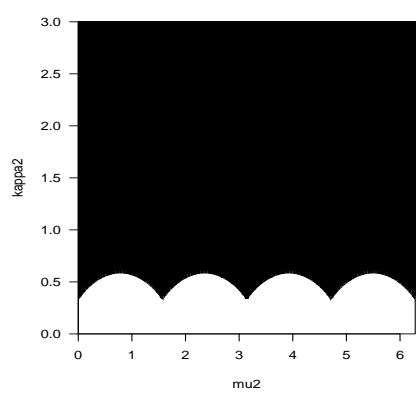

(a)

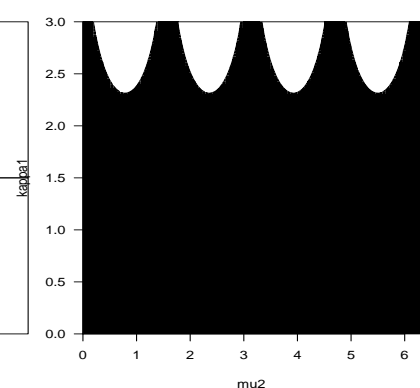

(b)

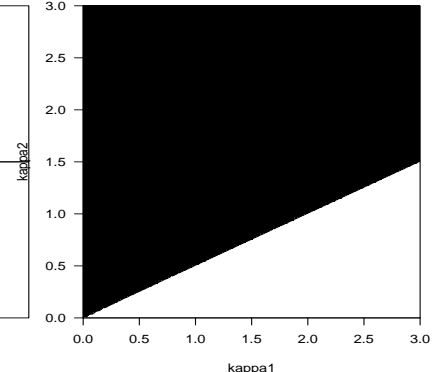

(c)

Figure 11: Discriminant (6) for the generalised von Mises density with $\mu_{1}=0$ as functions of: (a) $\left(\mu_{2}, \kappa_{2}\right)$ with $\kappa_{1}=1.16$, (b) $\left(\mu_{2}, \kappa_{1}\right)$ with $\kappa_{2}=1.16$, and (c) $\left(\kappa_{1}, \kappa_{2}\right)$ with $\mu_{2}=\pi / 4$. The region of positive discriminant/bimodality is displayed in black while the region of negative discriminant/unimodality is exhibited in white.

and Jammalamadaka (2007) show that the condition determining the unimodality or otherwise of the gvM distribution can also be reduced to the behaviour of the roots of a quartic equation. We apply the change of variable $x=\tan (\theta / 2)$, which is also used in Yfantis and Borgman (1982), so that the real roots of our quartic equation can take any value, and hence that discriminant (6) applies. Formula (5) then arises with

$b_{0}=b_{4}=-\sin \delta \cos \delta, \quad b_{1}=2\left(\rho+1-2 \sin ^{2} \delta\right), \quad b_{2}=6 \sin \delta \cos \delta, \quad b_{3}=2\left(\rho-1+2 \sin ^{2} \delta\right) ;$

here, $\delta=\left(\mu_{1}-\mu_{2}\right) \bmod \pi, \rho=\kappa_{1} /\left(4 \kappa_{2}\right)$. For comparison with Figure 1 associated with density (2), we plot positive (bimodal density) and negative (unimodal density) areas of the discriminant $D$ arising from (15) in Figure 11. We see a much greater proportion of bimodal distributions in the gvM family than in family (2). By the way, the frame corresponding to Figure 11(c) is absent from Figure 1 because it is blank. There, all densities are unimodal for the corresponding values of $\kappa$ and $r$; here, densities are unimodal (when $\mu_{2}=\pi / 4$ ) only when $\kappa_{1}>2 \kappa_{2}$.

This degree of bimodality is a two-edged sword. It is advantageous when bimodality is expected (as it often will be) from a process or situation involving two groups 
with locations $\mu_{1}$ and $\mu_{2}$. This is the same argument that leads to consideration of two-component mixture distributions (of, for example, von Mises distributions) but Gatto and Jammalamadaka (2007, p.352) argue, not unreasonably, that the gvM may have advantages over the mixture alternative in such situations. On the other hand, in more purely empirical situations in which there is no particular reason to expect, or evidence for, bimodality but there is evidence of asymmetry, the greater proportion of asymmetric unimodal distributions in family (2) seems rather more attractive.

\section{DISCUSSION}

Our work on distribution (2) was motivated by our interest in statistical uses of the Möbius transformation and, in particular, by initially seeking an asymmetric extension of the Jones and Pewsey (2005) class of distributions. Applying the Möbius transformation to the random variable $\hat{\theta}$ following the Jones and Pewsey distribution with $\mu=0$ results in a new family of distributions with density proportional to

$$
f_{G J P}(\theta) \propto \frac{\{1+\xi \cos (\theta-\eta)\}^{1 / \psi}}{\left\{1+r^{2}-2 r \cos (\theta-\nu)\right\}^{1 / \psi+1}}, \quad 0 \leq \theta<2 \pi
$$

where $\xi \geq 0$ and $0 \leq \eta<2 \pi$ satisfy

$\xi \cos \eta=\frac{\left\{r^{2} \cos (2 \nu)+1\right\} \tanh (\kappa \psi)-2 r \cos \nu}{1+r^{2}-2 r \cos \nu \tanh (\kappa \psi)} \quad$ and $\quad \xi \sin \eta=\frac{r^{2} \sin (2 \nu) \tanh (\kappa \psi)-2 r \sin \nu}{1+r^{2}-2 r \cos \nu \tanh (\kappa \psi)}$.

The normalizing constant of (16) is given by

$$
\frac{\left(1-r^{2}\right)\left\{\left(1+r^{2}\right) \cosh (\kappa \psi)-2 r \cos \nu \sinh (\kappa \psi)\right\}^{1 / \psi}}{2 \pi P_{1 / \psi}\{\cosh (\kappa \psi)\}} .
$$

Trivially, $r=0$ yields Jones and Pewsey's (2005) distribution (in which case, the parameter $\nu$ is also redundant). Also, the family (16) is symmetric if the parameters are chosen such that $\eta= \pm \nu$. This four-parameter symmetric family is considered in detail by Abe, Shimizu and Pewsey (2008). The full family (16) contains five parameters. On grounds of parsimony and interpretability, we prefer to pursue four-, or even three-, parameter distribution families that also allow skewness and that, hopefully, are just as useful, if not more so, in practice. (By the same token, we prefer a three-parameter family of symmetric distributions.) To those ends, we have 
concentrated in this paper on the subfamily of (16) corresponding to its limit as $\psi$ goes to 0 , and further subfamilies thereof.

Formulation (2), and its sub-families (7) and (13), has availed itself of a large number of interesting properties and features which have been described in this paper. We feel that it has considerable potential as a generally useful family of distributions for modelling skewness and varying 'tailweights' in circular data. We have slight misgivings about the bimodality of these densities for certain parameter configurations, but are relatively content that bimodality occurs in only a minority of cases.

Central to this work has been the use of the Möbius transformation. It is particularly advantageous to adopt our family as an angular error in circular-circular regression because of the connection between the proposed family and the regression curve whenever the latter is itself appropriate to formulate as a Möbius transformation.

\section{APPENDIX: ELEMENTS OF PROOFS}

\section{A.1 Proof of Theorem 1}

Clearly, the density (2) is symmetric if $r=0, \nu=0, \pi$ or $\kappa=0$. We consider the necessary condition for symmetry. Let $f$ be the density (2) and assume that $r \neq 0, \nu \neq 0, \pi$, and $\kappa \neq 0$. The density $f$ is said to be symmetric if there exists a constant $0 \leq a<\pi$ such that

$$
f(\mu+\nu+a-\theta)=f(\mu+\nu+a+\theta) \quad \text { for all } 0 \leq \theta<2 \pi
$$

It is clear that the following holds

$$
\begin{aligned}
& f(\mu+\nu+a-\theta)-f(\mu+\nu+a+\theta) \\
& \quad=\frac{1-r^{2}}{2 \pi \mathcal{I}_{0}(\kappa)} \exp \left[\frac{\kappa\{\xi \cos (\mu+\nu+a-\theta-\eta)-2 r \cos \nu\}}{1+r^{2}-2 r \cos (\theta-a)}\right] \frac{1-A(\theta)}{1+r^{2}-2 r \cos (\theta-a)}
\end{aligned}
$$

where

$$
\begin{aligned}
A(\theta)= & \exp \left\{\frac{\kappa\{\xi \cos (\mu+\nu+a+\theta-\eta)-2 r \cos \nu\}}{1+r^{2}-2 r \cos (\theta+a)}-\frac{\kappa\{\xi \cos (\mu+\nu+a-\theta-\eta)-2 r \cos \nu\}}{1+r^{2}-2 r \cos (\theta-a)}\right\} \\
& \times \frac{1+r^{2}-2 r \cos (\theta-a)}{1+r^{2}-2 r \cos (\theta+a)} .
\end{aligned}
$$


If $f$ is symmetric, then $A(\theta)=1$ for all $0 \leq \theta<2 \pi$. We show that

$$
\begin{gathered}
\kappa\left\{\frac{\xi \cos (\mu+\nu+a+\theta-\eta)-2 r \cos \nu}{1+r^{2}-2 r \cos (\theta+a)}-\frac{\xi \cos (\mu+\nu+a-\theta-\eta)-2 r \cos \nu}{1+r^{2}-2 r \cos (\theta-a)}\right\} \\
+\log \left\{\frac{1+r^{2}-2 r \cos (\theta-a)}{1+r^{2}-2 r \cos (\theta+a)}\right\}
\end{gathered}
$$

is not equal to 0 for some $0 \leq \theta<2 \pi$. The second term of the above implies that $a$ should be 0 or $\pi$ since $r \neq 0$. Because $\kappa \neq 0$, it follows that

$$
B(\theta) \equiv \frac{\xi \cos (\mu+\nu+a+\theta-\eta)-2 r \cos \nu}{1+r^{2}-2 r \cos (\theta+a)}-\frac{\xi \cos (\mu+\nu+a-\theta-\eta)-2 r \cos \nu}{1+r^{2}-2 r \cos (\theta-a)}
$$

should be 0 for any $0 \leq \theta<2 \pi$. When $a=0, B(\theta)$ can be expressed, after some manipulation, as

$$
B(\theta)=-\frac{\left(1-r^{2}\right) \sin \nu \sin \theta}{1+r^{2}-2 r \cos \theta} .
$$

Since $\nu \neq 0, \pi, B(\theta)$ is not equal to 0 for some $\theta$. Similarly, $B(\theta) \neq 0$ for some $\theta$ for $a=\pi$. Therefore $f$ is not symmetric.

\section{A.2 Proof of Theorem 2}

Making the successive substitutions $\cos \phi=\left\{\left(1+r^{2}\right) \cos \theta-2 r\right\} /\left(1+r^{2}-2 r \cos \theta\right)$ and then $x=\frac{1}{2}(\cos \phi+1)$, one obtains

$$
\begin{aligned}
E\left(\cos ^{n} \Theta\right)= & \frac{1}{\pi \mathcal{I}_{0}(\kappa)} \int_{0}^{\pi} \cos ^{n} \theta \exp \left[\frac{\kappa\left\{\left(1+r^{2}\right) \cos \theta-2 r\right\}}{1+r^{2}-2 r \cos \theta}\right] \frac{\left(1-r^{2}\right) d \theta}{1+r^{2}-2 r \cos \theta} \\
= & \frac{1}{\pi \mathcal{I}_{1}(\kappa)} \int_{0}^{\pi}\left\{\frac{\left(r^{2}+1\right) \cos \phi+2 r}{2 r \cos \phi+r^{2}+1}\right\}^{n} \exp \{\kappa \cos \phi\} d \phi \\
= & \frac{1}{\pi \mathcal{I}_{0}(\kappa)} \int_{0}^{1}\left\{\frac{\left(r^{2}+1\right)(2 x-1)+2 r}{2 r(2 x-1)+r^{2}+1}\right\}^{n} \exp \{\kappa(2 x-1)\} \frac{d x}{\sqrt{x(1-x)}} \\
= & \frac{e^{-\kappa}}{\pi \mathcal{I}_{0}(\kappa)} \int_{0}^{1}\left\{-1+\frac{2\left(1+r^{2}\right)}{(1-r)^{2}} x\right\}^{n}\left\{1+\frac{4 r}{\left(1-r^{2}\right)^{2}} x\right\}^{-n} \frac{e^{2 \kappa x}}{\sqrt{x(1-x)}} d x \\
= & \frac{e^{-\kappa}}{\pi \mathcal{I}_{0}(\kappa)} \sum_{j=0}^{n}\left(\begin{array}{l}
n \\
j
\end{array}\right)(-1)^{j}\left\{\frac{2\left(1+r^{2}\right)}{(1-r)^{2}}\right\}^{n-j} \\
& \times \int_{0}^{1}\left\{1+\frac{4 r}{(1-r)^{2}} x\right\}^{-n} x^{-\frac{1}{2}+n-j}(1-x)^{-\frac{1}{2}} e^{2 \kappa x} d x .
\end{aligned}
$$

Using (3.385) of Gradshteyn and Ryzhik (1994), we have

$$
\begin{aligned}
E\left(\cos ^{n} \Theta\right)= & \frac{e^{-\kappa}}{\pi \mathcal{I}_{0}(\kappa)} \sum_{j=0}^{n}\left(\begin{array}{c}
n \\
j
\end{array}\right)(-1)^{j}\left\{\frac{2\left(1+r^{2}\right)}{(1-r)^{2}}\right\}^{n-j} B\left(\frac{1}{2}+n-j, \frac{1}{2}\right) \\
& \times \Phi_{1}\left(\frac{1}{2}+n-j, n, n-j+1,-\frac{4 r}{(1-r)^{2}}, 2 \kappa\right) .
\end{aligned}
$$




\section{A.3 Formulae for Calculation of Expectations of (8) and (9)}

To evaluate the expectations of terms in (8) and (9), we make the first substitution of Appendix A.2 to transform back to a problem involving trigonometric moments of the von Mises distribution. In particular, we find that, if $\Theta \sim(7)$ with $\mu=0$, $B(\Theta)=1+r^{2}-2 r \cos \Theta$ and $\Phi \sim \operatorname{vM}(0, \kappa)$, then

$$
\begin{gathered}
E\left[B^{-2}(\Theta)\left\{\sin ^{2} \Theta-(r-\cos \Theta)^{2}\right\}\right]=\left(1-r^{2}\right)^{-2}\left\{1-r^{2}-2 E\left(r \cos \Phi+\cos ^{2} \Phi\right)\right\}, \\
E\left[B^{-3}(\Theta)\left\{\sin ^{2} \Theta\left(2 \cos \Theta-3 r+r^{3}\right)\right\}\right]=\left(1-r^{2}\right)^{-2} E\left\{\sin ^{2} \Phi(r+2 \cos \Phi)\right\}
\end{gathered}
$$

and

$$
E\left(B^{-2}(\Theta) \sin ^{2} \Theta\right)=\left(1-r^{2}\right)^{-2} E\left(\sin ^{2} \Phi\right)
$$

To complete the calculations, use the facts that $E\{\cos (n \Phi)\}=\mathcal{I}_{n}(\kappa) / \mathcal{I}_{0}(\kappa)$ (Mardia and Jupp, 1999, (3.5.29)) and the relationship $\kappa\left\{\mathcal{I}_{\nu-1}(\kappa)-\mathcal{I}_{\nu+1}(\kappa)\right\}=2 \nu \mathcal{I}_{\nu}(\kappa)$ (Gradshteyn and Ryzhik, 1994, (8.486.1)).

\section{REFERENCES}

Abe, T., Shimizu, K., and Pewsey, A. (2008), "Symmetric Unimodal Models for Directional Data Motivated by Inverse Stereographic Projection," under consideration.

Best, D.J., and Fisher, N.I. (1979), "Efficient Simulation of the von Mises Distribution," Applied Statistics, 28, 152-157.

Cox, D.R., and Reid, N.I. (1987), "Parameter Orthogonality and Approximate Conditional Inference," (with discussion) Journal of the Royal Statistical Society Series $B, 49,1-39$.

Downs, T.D., and Mardia, K.V. (2002), "Circular Regression," Biometrika, 89, 683697.

Fisher, N.I. (1993), Statistical Analysis of Circular Data, Cambridge: Cambridge University Press. 
Gatto, R., and Jammalamadaka, S.R. (2003), "Inference for Wrapped Symmetric $\alpha$-Stable Circular Models," Sankhyā, 65, 333-355.

Gatto, R., and Jammalamadaka, S.R. (2007), "The Generalized von Mises Distribution," Statistical Methodology, 4, 341-353.

Gradshteyn, I.S., and Ryzhik, I.M. (1994), Table of Integrals, Series, and Products, 5th ed., San Diego: Academic Press.

Hill, G.W. (1977), "Algorithm 518. Incomplete Bessel Function $\mathcal{I}_{0}$ : the von Mises Distribution," ACM Transactions on Mathematical Software, 3, 279-284.

Jammalamadaka, S.R., and SenGupta, A. (2001), Topics in Circular Statistics, Singapore: World Scientific.

Jones, M.C., and Pewsey, A. (2005), "A Family of Symmetric Distributions on the Circle," Journal of the American Statistical Association, 100, 1422-1428.

Kato, S., Shimizu, K., and Shieh, G.S. (2008), "A Circular-Circular Regression Model," Statistica Sinica, 18, 633-645.

Mardia, K.V. (1972), Statistics of Directional Data, London: Academic Press.

Mardia, K.V., and Jupp, P.E. (1999), Directional Statistics, Chichester, Wiley.

Maksimov, V.M. (1967), "Necessary and Sufficient Conditions for the Family of Shifts of Probability Distributions on Continuous Bicompact Groups," (in Russian) Theoria Verojatna, 12, 307-321.

McCullagh, P. (1996), "Möbius Transformation and Cauchy Parameter Estimation," Annals of Statistics, 24, 787-808.

Pewsey, A. (2002), "Testing Circular Symmetry," Canadian Journal of Statistics, 30, 591-600.

Pewsey, A., Lewis, T., and Jones, M.C. (2007), "The Wrapped $t$ Family of Circular Distributions," Australian and New Zealand Journal of Statistics, 49, 79-91. 
Rudin, W. (1987), Real and Complex Analysis, 3rd ed., New York: McGraw-Hill.

SenGupta, S., and Rao, J.S. (1966), "Statistical Analysis of Crossbedding Azimuths from the Kamthi Formation Around Bheemaran, Pranhati-Godavari Valley," Sankhyā Series B, 28, 165-174.

Uspensky, J.V. (1948), Theory of Equations, New York: McGraw-Hill.

Yfantis, E.A., and Borgman, L.E. (1982), "An Extension of the von Mises Distribution," Communications in Statistics - Theory and Methods, 11, 1695-1706. 